\title{
Locomotor Illusions Can Arise from Perceiving Multisensory Stimuli Configuring into a Sensorimotor Body-Environment Structure
}

Martin Dobricki, ${ }^{1,2 *}$ David Weibel, ${ }^{1}$ Leonardo Angelini, ${ }^{3}$ Elena Mugellini ${ }^{3}$ \& Fred W. Mast ${ }^{1}$

${ }^{1}$ University of Bern, 3012 Bern, Switzerland

${ }^{2}$ Swiss Federal Institute for Vocational Education \& Training, 3052 Zollikofen, Switzerland

${ }^{3}$ University of Applied Sciences of Western Switzerland, 1705 Fribourg, Switzerland

${ }^{*}$ Correspondence: martin.dobricki@ehb.swiss

\begin{abstract}
Walking through an environment involves perceiving one's walking body and its environment relative to each other. The sensation of placing one foot in front of the other may therefore arise from sensory stimuli configuring into the structure of a body perambulating an environment. Sensory stimuli constituting such a locomotor structure may accordingly bear the potential to induce in seated individuals the locomotor illusion of walking through an environment. To date, this was not investigated. Here, we have therefore exposed sixteen seated human subjects in a life-sized virtual environment to visual and tactile stimuli that configured either into the structure of a body perambulating an environment or into a control structure. Our subjects experienced locomotor illusions exclusively when the visuo-tactile stimuli configured into the "body-perambulating-environment" structure. This suggests that the locomotor illusion of walking through an environment can arise in seated individuals from perceiving sensory stimuli configuring into such a sensorimotor body-environment structure.
\end{abstract}

Keywords: walking, kinesthesia, perceptual processing, motor illusion

\section{Introduction}

Walking through an environment requires to perceive one's walking body and its environment relative to each other (Dobricki \& Pauli, 2016). The brain may process the sensory input related to such motor action (Wolpert $\&$ Flanagan, 2001) also relative to each other. The experience of locomotor 
behavior might therefore arise from perceiving sensory stimuli configuring (Wagemans et al., 2012) into the sensorimotor structure of a body perambulating an environment. Sensory stimuli constituting such a locomotor structure may accordingly induce in seated individuals the illusion of walking through an environment. To date, this was not investigated.

It was already attempted in previous studies to induce the illusion of walking in seated individuals with sensory stimuli (Amemiya et al., 2019; Kitazaki et al., 2016; Kokkinara et al., 2016; Terziman et al., 2012; Turchet et al., 2013). However, neither of such studies had considered the sensorimotor structure into which their stimuli were configuring. Neither of them had, moreover, examined whether the experience induced by their stimuli was featuring the kinesthesia (Proske \& Gandevia, 2009) of placing one foot in front of the other. Hence, the illusion induced by the stimuli of these studies was possibly that of walking in place (Whitton \& Peck, 2013) or that of controlling the walking movements of a virtual body (Kokkinara et al., 2016) devoid of any kinesthesia. It is accordingly an open question if the illusion of walking through an environment can arise from sensory stimuli configuring into a locomotor structure. Here, we have therefore exposed seated human subjects to stimuli eliciting the visual input known to result from locomotor behavior together with tactile stimuli applied to their foot soles. We presented the visuo-tactile stimuli in a life-sized virtual environment such that they constituted either a locomotor structure or a control structure. We predicted that exclusively the stimuli with the locomotor structure could induce the locomotor illusion of walking through an environment. In line with this prediction we also expected these stimuli to induce such an illusion independently of their load on the sensory system.

\section{Results}

We asked healthy human subjects to sit on a chair and to place their calves on another chair such that they could relax their legs. We then attached small vibratory boards to the sole of each of their feet (Fig. 1a). Wearing a head-mounted display, the subjects were enabled to view a life-sized virtual environment with some trees and a hill (Fig. 1b) from a first-person perspective that had the height of their eyes in an upright posture. Being exposed to this 
environment, the subjects' feet were stimulated with the vibratory devices while their virtual perspective was both swaying and moving forward like a walking human's head. Their perspective was first moved across a meadow for two minutes, and then moved up a hill (Fig. 1b) for another two minutes. During this motion the participants were not presented with a virtual body at any time.

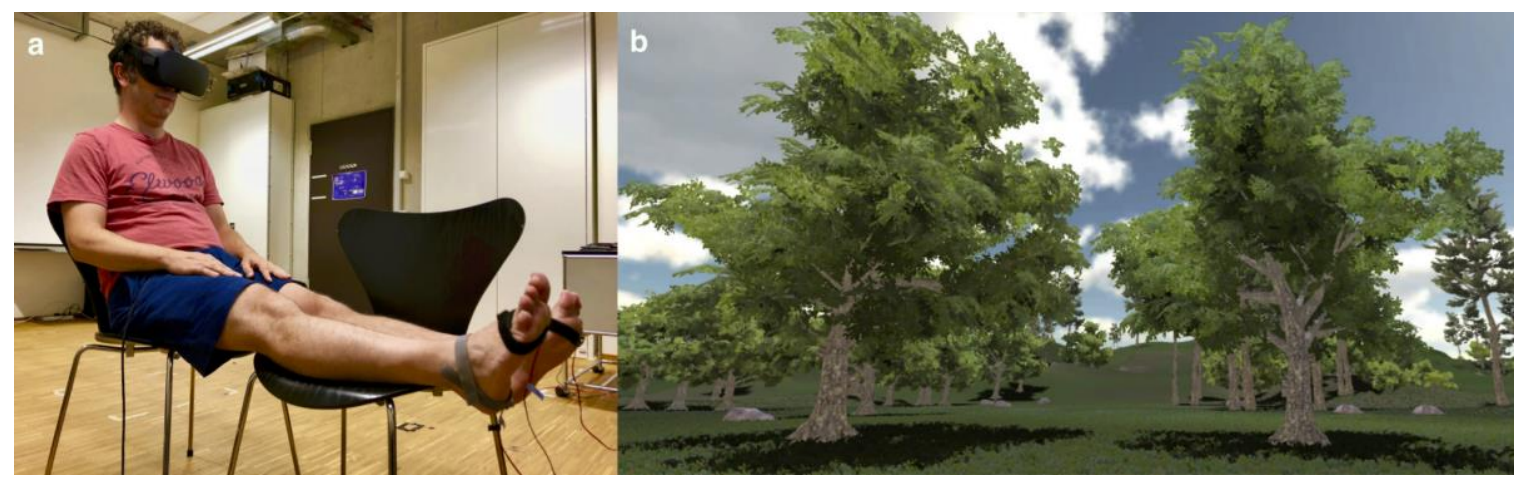

Figure 1. The experimental setup. (a) Seated male subject having two vibratory devices attached to the sole of each foot and wearing a head-mounted display that was used to enable the subject to experience (b) a life-sized 3D virtual environment in which his virtual perspective while swaying like the head of a walking human was first moving forward across a meadow and then up a hill.

We manipulated the "structure" to which the visuo-tactile stimuli could configure by switching the vibratory devices either constantly on both foot soles on (control structure), or alternately on one sole followed by the other on and off (locomotor structure). We manipulated the sensory "load" of the visuotactile stimuli by simulating the sensory input of locomoting either at a normal walking speed (normal load) or at a fast speed (augmented load). Following a within-subjects design, participants were exposed to each of the four possible combinations of the manipulation of the structure and the load of the visuotactile stimuli. At the end of each of these four trials, the participants were asked to take the head-mounted display off and to rate their subjective experience during the four minutes of a trial. For this purpose, we used a set of ten self-report statements presented with a continuous visual analogue scale $(\min =0 ; \max =100)$, which had its left pole labelled "not at all" and its right pole labelled "very much". One statement served to measure the participants' basic sensation of moving through space (see Methods). The 
following three statements on bipedal locomotion sensations served to measure locomotor illusions: I felt that I was walking. I had the impression to be placing one foot in front of the other. I felt that my legs were moving. The following three statements on locomotion sensations other than bipedal locomotion sensations served as control statements: I felt that I was sliding along the floor. I had the impression to be riding on a bicycle. I felt that I was being pushed on a wheelchair. In addition, the participants were asked to assess their "place illusion" of being located in the virtual environment (Slater, 2009) with three statements on sensations of spatial "presence" (see Methods).

We used the participants' mean rating of the three statements on bipedal locomotion, other locomotion, or spatial presence sensations as their overall rating scores in experiencing each of these sensation types. The rating scores of bipedal and other locomotion sensations served to examine if visuotactile stimuli configuring into a locomotor structure could induce locomotor illusions (see Methods). For checking if the hypothesized experiential effect of the "structure" of our stimuli was independent of their sensory "load" we have considered the interaction (see Methods) between these two factors.

In the trials with a locomotor stimulation structure the participants' rating of the locomotor illusion statement "I had the impression to be placing one foot in front of the other" was about medium, $M d=45.3$; IQR [30.5, 65.5], and it was significantly higher, $Z=-3.47 ; p=.001, r_{\text {contrast }}=.87$, than in the control trials, $M d=9.3$; IQR [2.0, 23.5]. This was also the case for their ratings of the other two locomotor illusion statements "I felt that my legs were moving", $Z=-3.21 ; p=.001, r_{\text {contrast }}=.80$, and "I felt that I was walking", $Z=-3.03 ; p=$ $.002, r_{\text {contrast }}=.76$.

The overall rating of locomotor illusions was about medium in the trials with a locomotor stimulation structure, $M d=45.1$; IQR [31.9, 59.1], and it was significantly higher, $Z=-3.36 ; p=.001, r_{\text {contrast }}=.84$, than in the control trials, $M d=13.7 ; \operatorname{IQR}[6.5,32.1]$. There was no significant interaction between the "structure" and the "load" of the visuo-tactile stimuli regarding the overall rating of locomotor illusions, $Z=-0.20, p=.836, r_{\text {contrast }}=.05$. The participants rated furthermore their basic sensation of moving through space to be approximately the same, $Z=-1.32, p=.187, r_{\text {contrast }}=.33$, in the trials with a 
locomotor stimulation structure, $M d=60.3$; IQR $[47.8,79.1]$ and in the control trials, $M d=55.5 ; \mathrm{IQR}[41.3,70.0]$.

As depicted in Figure 2, only in the trials with a locomotor stimulation structure was the overall rating of bipedal locomotion sensations, i.e., of locomotor illusions significantly higher, $Z=-3.51 ; p=.000, r_{\text {contrast }}=.87$, than the overall rating of the control statements on other locomotion sensations. The overall rating of the control statements was moreover significantly higher, $Z=-2.95 ; p=.003, r_{\text {contrast }}=.74$, in the control trials (see Fig. 2) than in the trials with a locomotor stimulation structure.

\section{Eipedal locomotion sensations 地 other locomotion sensations}

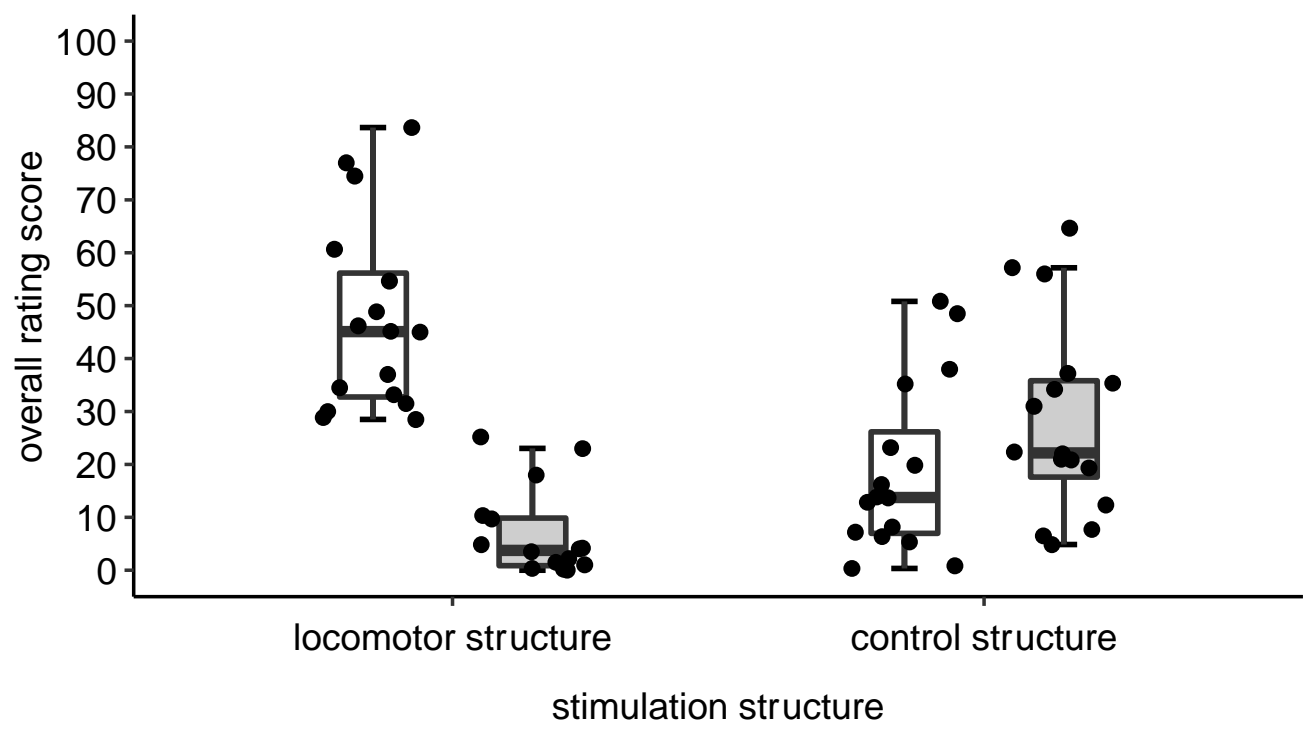

Figure 2. Comparison of the locomotor illusion and control ratings.

Box-and-whisker plots of the overall ratings of locomotor illusions, i.e., of bipedal locomotion sensations and of control statements on other locomotion sensations in the trials with a locomotor stimulation structure and in the control trials (control structure). Bold horizontal lines indicate median overall rating score, boxes indicate the lower quartile and upper quartile, whiskers indicate the farthest data points within 1.5 times of the lower quartile and upper quartile, respectively. The circles depict the individual overall rating scores of the 16 subjects.

Finally, the participants' overall rating of their place illusion was significantly, $Z=-2.02, p=.044, r_{\text {contrast }}=.51$, higher in the trials with a 
locomotor stimulation structure, $M d=58.8$; $\operatorname{IQR}[36.9,74.5]$, than in the control trials, $M d=46.5$; IQR [30.8, 67.2].

\section{Discussion}

We found seated human subjects exposed to visuo-tactile stimuli to experience more bipedal than other locomotion sensations, and thereby locomotor illusions exclusively when the stimuli were configuring into the structure of a body perambulating an environment. Hence, no locomotor illusions were experienced when our stimuli eliciting the visual input known to result from locomotor behavior were accompanied by tactile stimuli with which they did not configure into such a locomotor structure. Neither of this did depend on the sensory load of the visuo-tactile stimuli. Our findings thereby converge in indicating that the illusion of locomotor behavior can arise in seated individuals from perceiving sensory stimuli configuring into a locomotor structure. Supporting this we moreover found locomotor illusions to involve just like physical locomotor behavior (Usoh et al., 1999) an intensified illusion to be located in the environment (Slater, 2009) in which they occurred.

Walking can result in sensory input such as visual, kinesthetic or tactile input on one's walking body. Bipedal locomotion sensations may accordingly be understood to emerge due to the brain integrating this multisensory input to a unitary percept (Ernst \& Bülthoff, 2004; Welch \& Warren, 1980) of one's walking body. Our findings are in line with this understanding of multisensory perception. Yet, they also suggest that the sensory input from which bipedal locomotion sensations emerge is having the sensorimotor structure of a body perambulating an environment. Thus, our results indicate that the percept of one's walking body is but a constituent of this sensorimotor "bodyenvironment" structure (Dobricki \& Pauli, 2016) and thereby of a more comprehensive perceptual "human-environment" relation (Heft, 2001). This may be the reason why locomotor behavior was found to modulate both the experience of oneself relative to space (Usoh et al., 1999) as well as that of space relative to oneself (Noel et al., 2015).

Walking requires to control the movements that this motor behavior involves. The sensation of such motor control (Kannape et al., 2010) is 
understood to arise due to the brain matching actual sensory input (Blakemore et al., 1998) to the input that its locomotor representation predicts (Wolpert \& Flanagan, 2001). The actual sensory input used for this sensorimotor integration and the sensory input giving rise to sensations of bipedal locomotion should be the same. Hence, the sensorimotor bodyenvironment structure that we found to induce locomotor illusions might play a key role for the sensation of natural and artificial locomotor control (Kannape et al., 2010; Kokkinara et al., 2016) to emerge from sensorimotor integration.

Spinal cord injury patients attempting to move their paralyzed feet were found to use the same brain network as healthy subjects executing such movements (Hotz-Boendermaker et al., 2008). We found bipedal locomotion sensations to arise from sensory stimuli configuring into a sensorimotor bodyenvironment structure. The learning of sensorimotor structures is essential for the brain to adjust motor action (Braun et al., 2010). Hence, the following may be speculated: The brain might be able to learn to couple its sensorimotor representation of locomotor action with sensory stimuli configuring into a sensorimotor body-environment structure. Exposing spinal cord injury patients to such sensory stimuli while they attempt to walk might accordingly serve their brain to reorganize the neural pathways it is using for their locomotor behavior (Edgerton et al., 2008). This procedure may bear the potential to enhance invasive treatments of individuals with incomplete spinal cord injury (Formento et al., 2018). It may even serve as a non-invasive alternative (Harnie et al., 2019) to invasive treatments (Wagner et al., 2018) that can support these patients in relearning to walk.

The interaction between the sensory input on one's gait and spatial context was found to serve the brain to regulate exploratory behavior (Dobricki $\&$ Pauli, 2016). Such perceptual processing might involve the formation of the same kind of sensorimotor structure that we found to give rise to locomotor illusions. Hence, survival circuits (LeDoux, 2012) may be hypothesized to ensure bodily well-being by using such locomotor structures to regulate human exploratory behavior. 


\section{Methods}

Participants. Sixteen healthy human subjects ( 9 females, mean age $=25.1$ years, $\mathrm{SD}=2.6$ years) with normal or corrected-to-normal vision participated. The participants gave their written informed consent and could withdraw from the study at any time. The experimental procedure was approved by the ethics board of the faculty of human sciences of the University of Bern and was in accordance with the Declaration of Helsinki.

Stimuli and apparatus. Participants were asked to wear an "Oculus rift" head-mounted display. This HMD consists of dual OLED displays with a resolution of $1200 \times 1080$ pixels per eye displayed at $90 \mathrm{~Hz}$. It has a 94 horizontal and $93^{\circ}$ vertical field-of-view. The HMD served to present the participants with a life-sized 3D virtual environment with some trees and a hill (Fig. 1b) from a first-person perspective that had the height of their eyes in an upright posture. This was achieved by using the graphics engine Unity3D on an Asus Prime Z270-K computer with an NVIDIA GeForce GTX 1070 graphics card. During the exposure to the virtual environment, the virtual perspective was simulating the head sway of a walking human (Massaad et al., 2007; Sekiya et al., 1997; Woledge et al., 2005) while moving across a meadow for two minutes, and subsequently to the top of a hill for another two minutes. The perspective was moving either for $288 \mathrm{~m}$ at a normal walking speed of $1.2 \mathrm{~m}$ per sec, or for $432 \mathrm{~m}$ at a fast speed of $1.8 \mathrm{~m}$ per sec. The sway of the virtual perspective was programmed to occur within a period of $579 \mathrm{msec}$ in the normal speed trials, and $485 \mathrm{msec}$ in the fast speed trials. The peak-to-peak amplitude of the vertical perspective sway within this "step" time was on average $27.6 \mathrm{~mm}(\mathrm{SD}=3.6 \mathrm{~mm})$ in the normal speed trials and $36.0 \mathrm{~mm}(\mathrm{SD}=4.0 \mathrm{~mm})$ in the fast speed trials. The horizontal perspective sway amplitude was on average $8.5 \mathrm{~mm}(\mathrm{SD}=7.4 \mathrm{~mm})$ in all trials. During the exposure to the motion of the swaying virtual perspective the participants were furthermore stimulated with four LilyPad vibe boards controlled by an Arduino microcontroller of which two were attached to the sole of each foot (Fig. 1a). These vibratory devices were either all switched on constantly on both feet, or two of them for $80 \mathrm{msec}$ alternately on one foot followed by the other foot, when the minimum of a vertical sway of the virtual perspective was 
reached. White noise was presented over headphones in order to mask any external noise.

Experimental design. Due to the differential tactile stimulation described in the previous section the visuo-tactile stimuli constituted either the "locomotor" structure of a body perambulating an environment or a "control" structure (factor 1: stimulation structure). In addition to this manipulation of the structure of the visuo-tactile stimuli, their load on the sensory system was manipulated (factor 2: sensory load). It was either "normal" or "augmented", as the visuo-tactile stimuli were eliciting the sensory input that would result from locomoting either at a normal speed or at a fast walking speed. There were accordingly four possible experimental combinations of the structure and the sensory load of the visuo-tactile stimuli. All participants were exposed to each of these combinations in the framework of a $2 \times 2$ within-subjects design. The order of these four trials was determined by a balanced Latin square. Participants were not presented with a virtual body (Kokkinara et al., 2016) in any of the four trials.

Procedure. First, the eye height of the participants was determined by asking them to stand in an upright position and to wear the HMD. Second, they were asked to sit on a chair and to place their calves on another chair (Fig. 1a) such that they could relax their legs. Third, the experimenter attached the vibratory boards to the sole of each of their feet. Subsequently, the participants were exposed for four minutes to each of the four experimental trials in which the visuo-tactile stimulation described above occurred. At the end of each trial the participants were asked to take off the HMD and to rate their subjective experience as described next. After finishing these ratings, they were asked to make a break of approximately two min.

Psychometric ratings. The participants assessed their subjective experience by rating a set of ten self-report statements. These statements were presented in a random order on a computer screen using an internet platform (www.soscisurvey.de). A visual analogue scale (VAS; $\min =0 ; \max =100$ ) was presented to the right of each statement. The VAS was a continuous horizontal line of about $4 \mathrm{~cm}$ with the left pole labelled "not at all", and the right pole labelled "very much". The participants were instructed to move a small vertical line on the VAS to rate the intensity of the experience described in each 
statement by using a computer mouse. One self-report statement was on the basic sensation of moving through space ("I felt that I was moving through space”). The following three statements on bipedal locomotion sensations (Cronbach's $\alpha=$.93) served to measure locomotor illusions: I felt that I was walking. I had the impression to be placing one foot in front of the other. I felt that my legs were moving. The following statements on locomotion sensations other than bipedal locomotion sensations served as control statements (Cronbach's $\alpha=.66$ ): I felt that I was sliding along the floor. I had the impression to be riding on a bicycle. I felt that I was being pushed on a wheelchair. The statements "I felt that I was walking" and "I felt that I was sliding along the floor" were adapted from previous studies (Amemiya et al., 2019; Kokkinara et al., 2016; Terziman et al., 2012). The other statements on bipedal and other locomotion sensations were newly formulated for the purpose of this study. In addition, the participants assessed their "place illusion" to be located in the virtual environment (Slater, 2009) with the following three statements on spatial "presence" sensations (Cronbach's $\alpha=$ .98) adapted from the "MEC Spatial Presence" questionnaire (Rössler, 2011): I felt like I was actually there in the virtual environment. It was as though my true location had shifted into the virtual environment. It seemed as though I was present in the virtual environment.

Statistical analysis. Due to their distribution the psychometric ratings were compared with the Wilcoxon signed rank test (two-tailed). For these comparisons a priori power analysis $\left(\mathrm{d}_{z} \approx .8, \alpha\right.$ error $=.05$, power $\left.=.8\right)$ suggested to examine sixteen subjects (Cohen, 1988). In addition to the Wilcoxon tests the effect size $r_{\text {contrast }}$ was calculated (Rosnow \& Rosenthal, 2003). As the descriptive statistics we calculated for all ratings their median $(M d)$ and interquartile range [IQR]. The power analysis was performed with the statistical software $\mathrm{G}^{*}$ Power. The other statistical analyses were performed with Microsoft Excel and the statistical software R. The visualization of the statistical results was generated with the ggplot2 package within the statistical software R. It consisted in box-and-whisker plots depicting also the individual overall ratings of the sixteen subjects as circles. 
The procedure to examine if visuo-tactile stimuli configuring into a locomotor structure can induce locomotor illusions was as follows: First, we have compared the participants' individual mean rating of each of the three statements on bipedal locomotion sensations (see previous section) in the trials with a locomotor stimulation structure with that in the control trials (see experimental design). Second, we calculated the participants' overall rating of locomotor illusions. We accomplished this by calculating their mean rating of the three statements on bipedal locomotion sensations (see previous section) in the trials with a locomotor stimulation structure and in the control trials. Subsequently, we compared these two overall ratings of locomotor illusions. Third, we calculated the participants' overall rating of the control statements. We accomplished this by calculating their mean rating of the three control statements on locomotion sensations other than bipedal locomotion sensations (see previous section) in the trials with a locomotor stimulation structure and in the control trials. Finally, we have compared the overall rating of bipedal locomotion sensations i.e., of locomotor illusions with the overall ratings of the control statements once in the trials with a locomotor stimulation structure and once in the control trials. In addition, we compared the participants' mean rating of their basic sensation of moving through space and overall rating of their place illusion (see previous section) in the trials with a locomotor stimulation structure with that in the control trials.

In order to explore if the hypothesized experiential effect of the "structure" of our stimuli was independent of their sensory "load" we have considered the interaction between these two experimental factors. We accomplished this as follows: First, the difference between the two levels of each factor (see experimental design) was calculated regarding the overall locomotor illusion rating. Subsequently, this difference was compared with

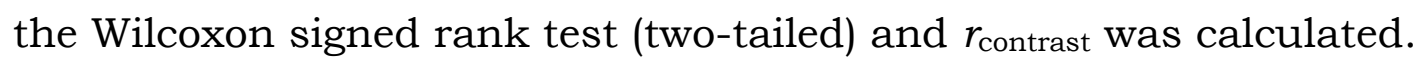

Data availability. The complete data set of this study along with a code-book are available at https://osf.io/pbnjq/

\section{Acknowledgments}

The authors would like to thank Dr. Maurizio Caon, Adrian Enderlin and Guillaume Pillonel for helping to prepare the technical setup, and Stephan Chen Xie for helping to run the experiments with human subjects. 


\section{References}

Amemiya, T., Ikei, Y., \& Kitazaki, M. (2019). Remapping peripersonal space by using foot-sole vibrations without any body movement.

Psychological Science, 30(10), 1522-1532.

https://doi.org/10.1177/0956797619869337

Blakemore, S. J., Wolpert, D. M., \& Frith, C. (1998). Central cancellation of self-produced tickle sensation. Nature Neuroscience, 1, 635-640. https://doi.org/10.1038/2870

Braun, D. A., Mehring, C., \& Wolpert, D. M. (2010). Structure learning in action. Behavioural Brain Research, 206, 157-165.

https://doi.org/10.1016/j.bbr.2009.08.031

Cohen, J. (1988). Statistical power analysis for the behavioral sciences (2nd ed.). Lawrence Erlbaum Associates.

Dobricki, M., \& Pauli, P. (2016). Sensorimotor body-environment interaction serves to regulate emotional experience and exploratory behavior.

Heliyon, 2, e00173. https://doi.org/10.1016/j.heliyon.2016.e00173

Edgerton, V. R., Courtine, G., Gerasimenko, Y. P., Lavrov, I., Ichiyama, R. M., Fong, A. J., Cai, L. L., Otoshi, C. K., Tillakaratnea, N. J. K., Burdick, J. W., \& Roy, R. R. (2008). Training locomotor networks. Brain Research Reviews, 57, 241-254.

https://doi.org/10.1016/j.brainresrev.2007.09.002

Ernst, M. O., \& Bülthoff, H. H. (2004). Merging the senses into a robust percept. Trends in Cognitive Sciences, 8(4), 162-169. https://doi.org/10.1016/j.tics.2004.02.002

Formento, E., Minassian, K., Wagner, F., Mignardot, J. B., Le GoffMignardot, C. G., Rowald, A., Bloch, J., Micera, S., Capogrosso, M., \& Courtine, G. (2018). Electrical spinal cord stimulation must preserve proprioception to enable locomotion in humans with spinal cord injury. Nature Neuroscience, 21(12), 1728-1741.

https://doi.org/10.1038/s41593-018-0262-6

Harnie, J., Doelman, A., de Vette, E., Audet, J., Desrochers, E., Gaudreault, N., \& Frigon, A. (2019). The recovery of standing and locomotion after spinal cord injury does not require task-specific training. eLife, 8, e50134. https://doi.org/10.7554/eLife.50134 
Heft, H. (2001). Ecological Psychology in context. Lawrence Erlbaum Associates.

Hotz-Boendermaker, S., Funk, M., Summers, P., Brugger, P., HeppReymond, M.-C., Curt, A., \& Kollias, S. S. (2008). Preservation of motor programs in paraplegics as demonstrated by attempted and imagined foot movements. NeuroImage, 39, 383-394. https://doi.org/10.1016/j.neuroimage.2007.07.065

Kannape, O. A., Schwabe, L., Tadi, T., \& Blanke, O. (2010). The limits of agency in walking humans. Neuropsychologia, 48, 1628-1636. https://doi.org/10.1016/j.neuropsychologia.2010.02.005

Kitazaki, M., Hirota, K., \& Ikei, Y. (2016). Minimal virtual reality system for virtual walking in a real scene. In S. Yamamoto (Ed.), Human Interface and the Management of Information. Lecture Notes in Computer Science (Vol. 9734, pp. 501-510). Springer. https://doi.org/10.1007/978-3$\underline{319-40349-6 \_48}$

Kokkinara, E., Kilteni, K., Blom, K. J., \& Slater, M. (2016). First person perspective of seated participants over a walking virtual body leads to illusory agency over the walking. Scientific Reports, 6, 28879. https://doi.org/10.1038/srep28879

LeDoux, J. (2012). Rethinking the emotional brain. Neuron, 73, 653-676. https://doi.org/10.1016/j.neuron.2012.02.004

Massaad, F., Lejeune, T. M., \& Detrembleur, C. (2007). The up and down bobbing of human walking: a compromise between muscle work and efficiency. The Journal of Physiology, 582, 789-799.

https://doi.org/10.1113/jphysiol.2007.127969

Noel, J. P., Grivaz, P., Marmaroli, P., Lissek, H., Blanke, O., \& Serino, A. (2015). Full body action remapping of peripersonal space: the case of walking. Neuropsychologia, 70, 375-384. https://doi.org/10.1016/j.neuropsychologia.2014.08.030

Proske, U., \& Gandevia, S. C. (2009). The kinaesthetic senses. The Journal of Physiology, 587(17), 4139-4146.

https://doi.org/10.1113/jphysiol.2009.175372 
Rosnow, L. R., \& Rosenthal, R. (2003). Effect sizes for experimenting psychologists. Canadian Journal of Experimental Psychology, 57(3), 221-237. https://doi.org/10.1037/h0087427

Rössler, P. (2011). Skalenhandbuch Kommunikationswissenschaft [Handbook of rating scales in communication science]. Springer VS.

Sekiya, N., Nagasaki, H., Ito, H., \& Furuna, T. (1997). Optimal walking in terms of variability in step length. Journal of Orthopaedic \& Sports Physical Therapy, 26, 266-268.

https://doi.org/10.2519/jospt.1997.26.5.266

Slater, M. (2009). Place illusion and plausibility can lead to realistic behaviour in immersive virtual environments. Philosophical Transactions of the Royal Society B, 364, 3549-3557. https://doi.org/10.1098/rstb.2009.0138

Terziman, L., Marchal, M., Multon, F., Arnaldi, B., \& Lécuyer, A. (2012). The king-kong effects: improving sensation of walking in VR with visual and tactile vibrations at each step. In Proceedings of the IEEE symposium on 3D user interfaces (pp. 19-26). IEEE. https://doi.org/10.1109/3DUI.2012.6184179

Turchet, L., Burelli, P., \& Serafin, S. (2013). Haptic feedback for enhancing realism of walking simulations. IEEE Transactions on Haptics, 6(1), 3545. https://doi.org/10.1109/TOH.2012.51

Usoh, M., Arthur, K., Whitton, M. C., Bastos, R., Steed, A., Slater, M., \& Brooks, F. P. (1999). Walking > walking-in-place $>$ flying, in virtual environments. In Proceedings of the 26th annual conference on computer graphics and interactive techniques (pp. 359-364). ACM. https://doi.org/10.1145/311535.311589

Wagemans, J., Feldman, J., Gepshtein, S., Kimichi, R., Pomerantz, J. R., van der Helm, P. A., \& van Leeuwen, C. (2012). A century of gestalt psychology in visual perception: II. conceptual and theoretical foundations. Psychological Bulletin, 138(6), 1218-1252. https://doi.org/10.1037/a0029334

Wagner, F. B., Mignardot, J.-B., Le Goff-Mignardot, C. G., Demesmaeker, R., Komi, S., Capogrosso, M., Rowald, A., Seáñez, I., Caban, M., Pirondini, E., Vat, M., McCracken, L. A., Heimgartner, R., Fodor, I., Watrin, A., 
Seguin, P., Paoles, E., Van Den Keybus, K., Eberle, G., Schurch, B., Pralong, E., Becce, F., Prior, J., Buse, N., Buschman, R., Neufeld, E., Kuster, N., Carda, S., von Zitzewitz, J., Delattre, V., Denison, T., Lambert, H., Minassian, K., Bloch, J., \& Courtine, G. (2018). Targeted neurotechnology restores walking in humans with spinal cord injury. Nature, 563(7729), 65-71. https://doi.org/10.1038/s41586-018-0649$\underline{2}$

Welch, R. B., \& Warren, D. H. (1980). Immediate perceptual response to intersensory discrepancy. Psychological Bulletin, 88, 638-667. https://doi.org/10.1037/0033-2909.88.3.638

Whitton, M. C., \& Peck, T. C. (2013). Stepping-driven locomotion interfaces. In F. Steinicke, Y. Visell, J. Campos, \& A. Lécuyer (Eds.), Human walking in virtual environments: perception, technology, and applications. Springer. https://doi.org/10.1007/978-1-4419-8432$\underline{6 \_11}$

Woledge, R. C., Birtles, D. B., \& Newham, D. J. (2005). The variable component of lateral body sway during walking in young and older humans. The journals of gerontology. Series A, Biological sciences and medical sciences, 60, 1463-1468.

https://doi.org/10.1093/gerona/60.11.1463

Wolpert, D. M., \& Flanagan, J. R. (2001). Motor prediction. Current Biology, 11(18), R729-R732. https://doi.org/10.1016/S0960-9822(01)00432-8 